\title{
Mediastinal mass: an unusual presentation of coccidioidomycosis
}

\author{
Imran Umer MD, Yasir Ahmed MD
}

\begin{abstract}
Coccidioidomycosis is an endemic fungal disease, found mainly in the southwestern United States, northwestern Mexico, and some areas of Brazil and Argentina. Clinical manifestations vary depending upon both the extent of infection and the immune status of the host. Pneumonia is the most common clinical presentation, and it rarely involves the central nervous system, skin, and bones. Patients with coccidioidomycosis usually respond well to therapy if diagnosed and treated promptly. Here we report a rare case of coccidioidomycosis infection in an immunocompromised host who presented with a mediastinal mass.
\end{abstract}

Key words: Coccidioidomycosis, Coccidioidomycosis immitis, fungal infection, mediastinal mass

\section{INTRODUCTION}

Coccidioidomycosis, the oldest of the major mycoses, was first recognized as a human disease in Argentina in 1892. It is a dimorphic fungus with distinct saprophytic yeast and parasitic mold stages. The Coccidioides genus has two species, Coccidioides immitis and Coccidioides posadasii. The species are genetically different but indistinguishable in terms of morphology and clinical presentations. ${ }^{2}$ Pneumonia is the most common clinical presentation. Rarely, disseminated infection, involving the skin, bones, joints, central nervous system, and other organs, can occur and has a high mortality rate. ${ }^{3}$ We are reporting a rare case of coccidioidomycosis infection presenting with a mediastinal mass.

Corresponding author: Yasir Ahmed MD

Contact Information: yasir.ahmed@ttuhsc.edu

DOI: 10.12746/swrccc2014.0206.075

\section{Case Presenttion}

A 35-year-old African American man, a chronic smoker with a longstanding human immunodeficiency virus (HIV) infection, presented with shortness of breath, low-grade fever, and dry cough for three days. He also had weight loss, headache, and dizziness. He denied night sweats, seizure, loss of consciousness, and skin rash. The patient stopped antiretroviral therapy one year prior to presentation and did not follow up with his physician during this period. His past medical history is significant for Pneumocystis jiroveci pneumonia (PJP), cytomegalovirus viremia, and bronchial asthma. Physical examination revealed a cachectic male in no distress. His pulse was 92 / minute, blood pressure $115 / 63 \mathrm{mmHg}$, respiratory rate 22 breaths/minute, $\mathrm{O}_{2}$ saturations $96 \%$ on room air, and temperature $100.9^{\circ} \mathrm{F}$. The rest of his physical examination was unremarkable.

The initial laboratory work up showed a CD4 count of $4 / \mu \mathrm{L}$, HIV viral load of 260,000 copies $/ \mathrm{ml}$, 


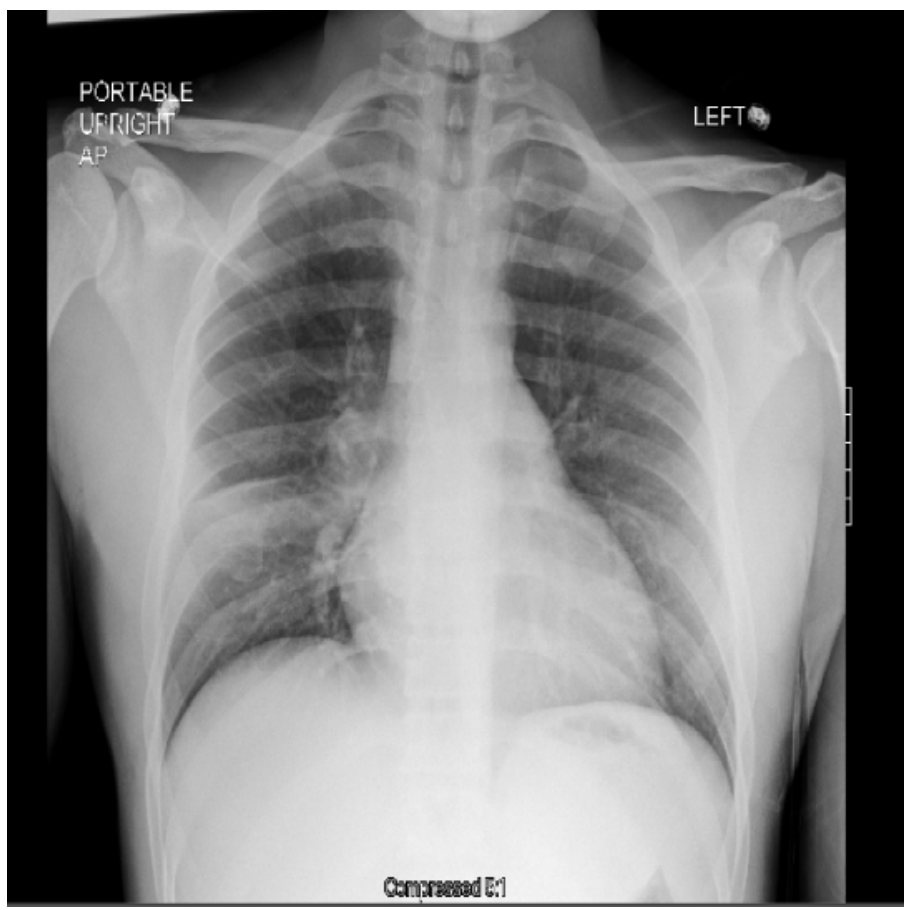

Figure 1: Chest X-ray AP view showing right middle lobe atelectasis or pneumonia.
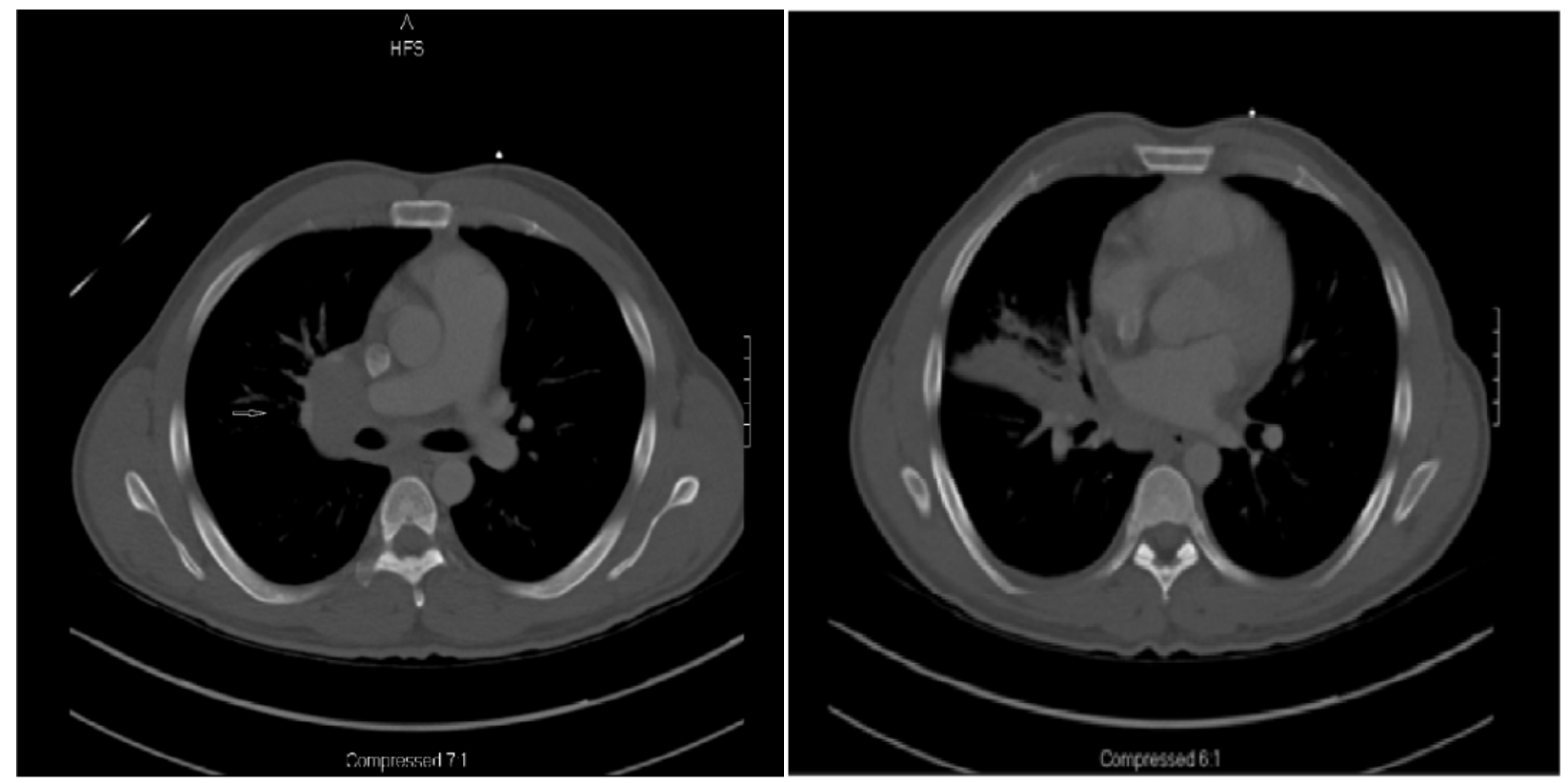

Figure 2a and 2b: Chest CT with contrast, mediastinal windows: arrow showing right mediastinal mass size $3 \times 4 \mathrm{~cm}$, also mild pretracheal lymphadenopathy with postobstructive atelectasis or pneumonia involving right middle lobe. 
culosis and Mycobacterium avium intracellulare (MAC) were negative. Magnetic resonance imaging of the brain showed diffuse mild cerebral volume loss and scattered white matter changes, consistent with HIV encephalopathy. Cerebrospinal fluid was negative for coccidioidomycosis antibody by complement fixation; other negative tests included VDRL, cryptococcal antigen, fungal cultures, acid fast bacillus culture, and $\mathrm{JC}$ virus by PCR. He was started on fluconazole 400 mg daily, azithromycin for MAC prophylaxis, and trimethoprim/sulfamethoxazole for PJP prophylaxis. His symptoms improved, and on hospital day ten he was discharged home on oral fluconazole $400 \mathrm{mg}$ daily, and PJP and MAC prophylaxis. Five weeks after discharge, a chest $x$-ray showed minimal improvement in the right middle lobe infiltrate. He was started on tenofovir, emtricitabine, and boosted darunavir for his HIV infection.

He was re-admitted seven weeks later with sepsis and worsening pulmonary infiltrates. A CT angiogram of the chest showed bilateral pulmonary infiltrates and a slight increase in size of the mediastinal mass. He required mechanical ventilation for severe hypoxic respiratory failure. Coccidioidomycosis immitis grew from the blood culture. The serum coccidioidomycosis antibody titers by complement fixation increased to 1:64, immunodiffusion was detected again, and coccidioidal serum IgG was high at 4.6. The family reported that the patient had stopped all medications five weeks prior to admission. The decision was made to place the patient on comfort care.

\section{Discussion}

Coccidioidomycosis is an endemic fungal disease mainly found in the Western Hemisphere, almost entirely between the latitudes of $40^{\circ}$ north and south. This life zone corresponds with the hot deserts of the southwestern United States and northwestern Mexico. In the US, this semiarid zone encompasses West Texas, Arizona, and parts of Nevada, Utah, New Mexico, and California. ${ }^{4}$ Risk factors for coccidioido- mycosis include extremes of age, living in or travel to an endemic area, immunosuppressed conditions, including the acquired immunodeficiency syndrome (AIDS), solid organ transplantation, pregnancy, and occupations that involve handling laboratory specimens from infected individuals. African Americans, Filipinos, and Hispanics are at a higher risk for disseminated disease. The route of exposure is primarily through inhalation of arthroconidia and rarely percutaneous infection by skin laceration.

Recently, the incidence of infection with coccidioidomycosis has risen to approximately 150, 000 per year in the US due to population increases in southern Arizona and central California. Sixty percent of these patients are asymptomatic, and the rest have presentations ranging from "flu-like" illness (approximately $15 \%$ ) to pneumonia (Valley fever) that becomes evident one to three weeks after infection. Such infections are usually indistinguishable from other respiratory infections and are usually self-limited. Five to ten percent of the cases result in residual pulmonary sequelae such as pulmonary nodules or peripheral thin-walled pulmonary cavities. Extrapulmonary disease involving the skin, bones, joints, central nervous system, and other organ systems are very uncommon, occurring $(<1 \%)$ in an immunocompetent host. Disseminated infection is more common in pregnancy, in immunocompromised hosts, such as patients with AIDS, lymphoma, solid organ transplants, and in patients receiving immunosuppressive or immunomodulating therapy.

The diagnosis of Coccidioidomycosis immitis infection is confirmed by identification of the fungus by culture of a tissue specimenor body fluid. Serological tests include enzyme-linked immunoassay assay (EIA), complement fixation (CF), and immunodiffusion (ID) are also useful in the diagnosis of coccidioidomycosis infections. Immunodiffusion and CF are the most specific methods for the diagnosis of coccidioidomycosis. Quantitative CF titer reflects the severity of the illness and provides a useful correlation with response to antifungal therapy. Serology can be negative, particularly in the early phase of the disease. 
Most patients with coccidioidomycosis infections are asymptomatic or have symptom resolution without antifungal treatment, especially in an immunocompetent host. Fluconazole is the drug of choice for symptomatic (>2 months duration symptoms) pulmonary and extra-pulmonary coccidioidomycosis infection. Treatment duration should be based on the site and extent of infection. Treatment details can be found elsewhere. ${ }^{6}$ Amphotericin is used for severe or disseminated infection and during pregnancy. Other azoles, especially posaconazole and voriconazole, are reserved for refractory cases as an alternative or salvage therapy. Surgical resection may be required for refractory well localized pulmonary nodules/or ruptured pulmonary cavities.

Our patient presented with a mediastinal mass, which is a very rare manifestation of coccidioidomycosis. Joshua et al reported a mediastinal mass in an immunocompetent patient, but in his case the patient also had interstitial infiltrate with pneumonia. Considering the history of AIDS, the differential diagnosis of the isolated mediastinal mass was very broad in our case and included chronic fungal infection, tuberculosis, atypical mycobacterial infection, lymphoma, and lung cancer. Evaluation confirmed coccidioidomycosis infection in our patient, but unfortunately, due to non-adherence with medications and an immunocompromised state, his infection became disseminated, resulting in respiratory failure and death.

In summary, coccidioidomycosis is an endemic fungus in West Texas, and the clinical manifestations are variable and non-specific. A mediastinal mass is a rare manifestation of coccidioidomycosis but should be considered in the differential diagnosis, especially in an immunocompromised host. An aggressive diagnostic approach including a biopsy should be employed promptly in such patients to rule out other infectious and malignant etiologies before starting antifungal therapy.
Author Affiliation: Imran Umer is a resident in Internal Medicine at TTUHSC in Permian Basin/Odessa, TX. Yasir Ahmed is an Assistant Professor Infectious Diseases, Internal Medicine at TTUHSC, Permian Basin/Odessa, TX.

Received: 02/26/2014

Accepted: 04/04/2014

Reviewers: Cynthia Jumper MD

Published electronically: 04/15/2014

Conflict of Interest Disclosures: none

\section{REFERENCES}

1. Posada A. Un nuevocaso de micosisfungoidea conn Psorospermias. Anales del Circulo Medico Argentino 1892; 15: 585-597.

2. Fisher MC, Koenig GL, White TJ, Taylor JW. Molecular and phenotypic description of Coccidioides posadasii sp. nov.previously recognized as the non-California population of Coccidioidesimmitis. Mycologia 2002 Jan-Feb; 94(1):73-84.

3. Saubolle MA, McKellar PP, Sussland D. Epidemiologic, clinical, and diagnostic aspects of coccidioidomycosis. J Clin Microbiol 2007;45(1):26-30.

4. Pappagianis G. Epidemiology of coccidioidomycosis. Curr Top Med Mycol 1988; 2: 199-238.

5. Ampel NM, Wieden MA, Galgiani JN. Coccidioidomycosis: clinical update. Rev Infect Dis 1989; 11:897-911.

6. Galgiani JN, Ampel NM, Blair JE. Catanzaro A, Johnson, RH, Stevens DA, Willimas PL. Coccidoidomycosis, IDSA guidelines . Clinical Infectious Diseases 2005;41:1217-23

7. Pappagianis D. Serologic studies in coccidioidomycosis. Semin Respir Infect 2001; 16:242-50.

8. Stephany JD, Lucero S, Walsh AF. Mediastinal Mass in a 27-Year-Old Man. Arch Pathol Lab Med 2005; 129(5):699-700. 